\title{
Accurate Coupling-centric Timing Analysis Incorporating Temporal and Functional Isolation
}

\author{
RAVISHANKAR ARUNACHALAM ${ }^{\mathrm{a}, *}$, RONALD DESHAWN BLANTON $^{\mathrm{b} \dagger}$ and LAWRENCE T. PILEGGI ${ }^{\mathrm{b}+}$ \\ ${ }^{\mathrm{a}}$ IBM Corporation, Mail Stop 904-6F015, 11400 Burnett Road, Austin, TX 78758, USA; ${ }^{\mathrm{b}}$ Department of Electrical and Computer Engineering, Carnegie \\ Mellon University, 5000 Forbes Avenue, Pittsburgh, PA 15213, USA
}

(Received 15 March 2001; Revised 30 January 2002)

\begin{abstract}
Neighboring line switching can contribute to a large portion of the delay of a line for today's deep submicron designs. The impact of this switching on delay is usually estimated by scaling the coupling capacitances (often by a factor of 2) and modeling them as grounded. This simple approach has been shown to be overly pessimistic in some cases, while somewhat optimistic in others. Apart from the delay modeling inaccuracies, the temporal and functional isolation of the aggressors can contribute to the pessimism. This paper introduces TACO, a timing analysis approach that addresses both these issues. TACO captures the provably worst-and best-case delays as a function of the timing-window inputs to the gates. We then present a comprehensive ATPG-based approach that uses functional information to identify valid interactions between coupled lines. Our algorithm accounts for glitches on aggressors that can be caused by static and dynamic hazards in the circuit. Results on industrial examples and benchmark circuits show the value of our approach.
\end{abstract}

Keywords: Timing analysis; Coupling; Logic information; Worst-case delay; Aggressor; Crosstalk

\section{INTRODUCTION}

Analyzing the impact of crosstalk on delay is critical for present day deep submicron circuits. When a coupled line switches in the opposite direction, the signal delay increases and when it switches in the same direction, the delay decreases. The affected line is referred to as the victim and the affecting line as the aggressor. A common technique used to compute worst-case delay (WCD) of the victim is to scale the coupling capacitances by a "scaling factor" of 2 and model them as grounded. While being overly pessimistic in some cases, this $2 \times$ approach has also been shown to underestimate the actual WCD in others [1], which is unacceptable. Moreover, this approach does not generalize to resistive interconnect, and it ignores signal switching times and the relative strengths of drivers. An accurate delay model that accounts for coupling must be able to address these issues. An upper bound of $3 \mathrm{X}$ for the scaling factor is presented in Refs. $[7,13]$, under certain assumptions.
The delay modeling problem forms only a subset of the problems posed by crosstalk to static timing analysis (STA). In order to impact the victim's delay, an aggressor has to switch simultaneously with the victim. There are two reasons why coupled lines may not switch togethertemporal isolation and functional isolation. In STA, timing characteristics are usually specified by arrival time windows, which represent the earliest and latest times that a signal can undergo a transition (either rising or falling). It is possible that a victim and an aggressor have arrival time windows that do not overlap, which implies that they cannot switch together. Functional isolation, on the other hand arises due to the logical relationships between gates. If the logic dictates, for example, that the victim and aggressor can never switch in opposite directions in the same clock cycle, then we have a "false coupling interaction." Ignoring temporal and functional isolation can lead to highly pessimistic results in STA.

In order to determine whether an aggressor and victim are temporally isolated, it is necessary to determine their

\footnotetext{
*Corresponding author. Tel.: +1-512-838-9454. Fax: + 1-512-838-4036. E-mail: ravaru@ austin.ibm.com

Tel.: +1-412-268-2987. Fax: + 1-412-268-6774. E-mail: blanton@ece.cmu.edu

Tel./Fax: + 1-412-268-6774. E-mail: pileggi@ece.cmu.edu
} 
switching windows first, but these in turn depend on the delays. Hence, we have a chicken-egg problem where the delays due to coupling and the switching windows that specify the arrival times are interdependent. The problem only gets compounded when multiple aggressors are involved. An iterative approach is presented in Ref. [6] and a non-iterative scheduling algorithm is presented in Ref. [11], but the delay model used is not accurate in either case.

In this paper, we present TACO, a crosstalk-centric timing analysis approach capable of handling coupling effects. In contrast to the scaling approach, TACO uses an accurate gate-delay engine to compute gate-delays in the presence of coupling. We use the model presented in Ref. [3] to determine WCD due to switching aggressors. We overcome the chicken-egg problem by starting with a worst case assumption for the switching windows. Under this assumption, the delay engine produces a pessimistic delay bound if the timing windows of the aggressors are not known a priori. The pessimism incurred is removed in subsequent iterations, in a provably convergent manner. Most importantly, we provide bounds on the delays and arrival times, which is very important in the context of STA.

It is important to determine the impact of functional isolation, even at the cost of extra CPU time, since it is unpredictable and largely design-dependent. A related problem encountered in STA is the well known false-path problem, which has been extensively researched. However, the problem of functional relationships of coupled signals is different in nature and has been addressed only recently for the noise problem [5]. In this paper, we present a comprehensive methodology to identify valid interactions between coupled lines, including a thorough analysis of glitches that can be caused by potential hazards. We use an ATPG approach with a six-valued algebra that includes static and dynamic hazards. Results are shown for benchmark circuits to consider the importance of modeling functional information to reduce the conservatism of most coupled delay models.

The rest of the paper is organized as follows. The second section describes the coupled delay model that we use and the aggressor alignment methodology to compute WCD. We formulate the coupled timing analysis problem in the third section, and describe the TACO approach. In the fourth section, we describe our ATPG approach for identifying functional isolation, and give static and dynamic sensitization criteria for valid coupling interactions. Conclusions and future work are presented in the fifth section.

\section{COUPLED DELAY MODEL}

The problem that needs to be solved, in the case of capacitive coupling, is shown in Fig. 1. There are $n$ aggressors switching along with the victim. Each aggressor is modeled as coupled only to the victim

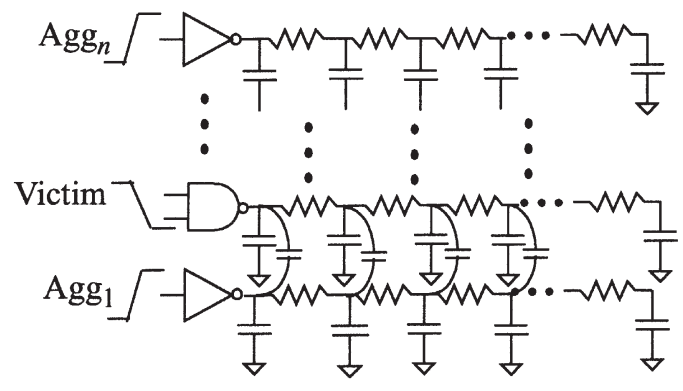

FIGURE $1 \quad \mathrm{~N}$ coupled lines switching simultaneously.

and coupled lines to aggressors are not included in the system. The coupling capacitances from aggressors to lines other than the victim are treated as grounded capacitances.

We use the coupled-gate effective capacitance (Ceff) model presented in Ref. [3] as the core delay engine. This engine uses the piecewise linear Thevenin equivalent model for the gate [2]. This model replaces the gate with a piecewise linear voltage source in series with a fixed driver resistance. The parameters of the voltage source (the waveform offset time, and the individual time intervals for the pieces) are pre-characterized and stored as a function of input transition time and load capacitance. Given the saturated input ramp signals to all the gates, the goal is to find the Thevenin equivalent model for each gate and then drive the multi-port circuit using these linear, time varying drivers.

For a single gate driving an RC interconnect, the $C_{\text {eff }}$ value (and hence the Thevenin model parameters) for the gate depends on the current drawn by the load. This current can be easily computed because the parameters of the load are known explicitly. But, when we have simultaneously switched coupled lines, each driver acts like a "load" to the other lines. The overall load current can be computed using superposition, as shown in Fig. 2. To avoid complexity of iterations, we perform the Ceff iterations for each gate in a decoupled manner. The interconnect is modeled by a coupled N-port, and PRIMA [10] or some similar scheme can be used to obtain a reduced-order macromodel for the N-port. The waveforms obtained from the model agree excellently with those obtained from HSPICE [3].

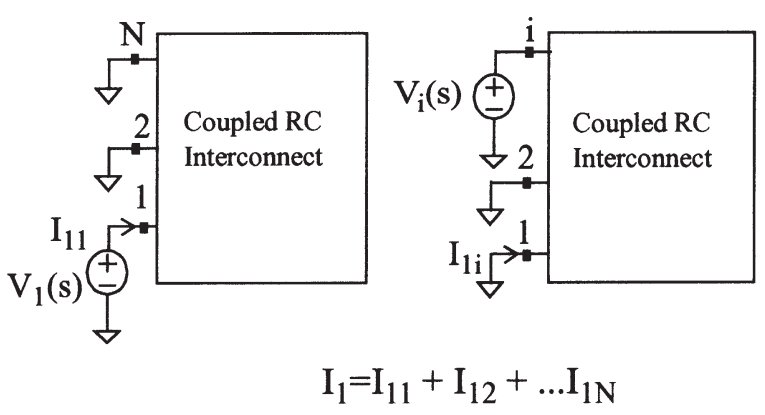

FIGURE 2 Calculation of load current by superposition. 
For computing best and worst case delays due to aggressor switching, it is necessary to align the aggressors with respect to the victim. This is performed as follows: first the noise waveform is computed, and the peak value of the noise $\mathrm{Vp}$ is found. This waveform is then aligned such that the peak occurs when the victim value is $\mathrm{Vdd} / 2-\mathrm{Vp}$ (for a falling victim), so as to just cause the victim to reach $\mathrm{Vdd} / 2$ again. The waveforms can be superposed within an iteration due to the linearized nature of the gate models used. This alignment process is explained in greater detail in Ref. [3].

\section{TACO}

The key ideas behind TACO can be stated as follows:

i) Perform STA such that the timing characteristics at aggressors can be used to determine if it can switch with the victim.

ii) If it can, determine the switching time of the aggressor such that it has maximum impact on the victim and bound the delays and arrival times.

We first define the terminology that will be used to describe our approach.

Input-output delay: This is defined with respect to a particular input node and fanout node of a gate, and is the time difference between the $50 \%$ point of the signal at the input and the $50 \%$ point of the signal at the fanout node.

Best-case delay $(B C D)$ : The input-output delay computed under the condition that the time at which the fanout node reaches the $50 \%$ point is minimized.

Worst-case delay: The input-output delay computed under the condition that the time at which the fanout node reaches the $50 \%$ point is maximized.

Aggressor net: A net that has "significant" coupling capacitance to the victim net so as to be able to influence the input-output delay of the victim gate. The criterion for coupling capacitance being "significant" is discussed in the "Pruning of aggressors" section.

Aligned aggressor: An aggressor is said to be an aligned aggressor with respect to a particular victim, if the switching window of the aggressor is such that it can affect to the BCD/WCD of the victim.

Early/Late arrival times (EAT/LAT): The EATs and LATs for fanout node $j$ of a gate with $n$ inputs are defined as follows:

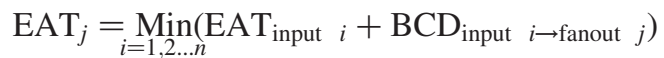

$$
\begin{aligned}
& \mathrm{LAT}_{j}=\underset{i=1,2 \ldots n}{\operatorname{Max}}\left(\mathrm{LAT}_{\text {input } i}+\mathrm{WCD}_{\text {input } i \rightarrow \text { fanout } j}\right)
\end{aligned}
$$

It must be noted that the EAT and LAT at the output can be determined by different inputs to the gate. The arrival times as given by Eqs. (1) and (2) are those obtained when performing block-based analysis. We choose block-based analysis for TACO because the arrival times at a node are then independent of the path propagated. To determine whether the aggressor can switch with the victim, we have to use the arrival time windows at the aggressor. In a pathbased analysis, we would not be able to conclude anything from the arrival time windows at the aggressor. The arrival times at the aggressor may not overlap with the victim's switching when the signal is propagated along a particular path, but may overlap for some other path. Since our goal is to obtain bounds on the delays and arrival times at the victim, we choose a block-oriented approach. We use the breadth-first search, whereby a gate in a particular level in the logic is processed only after all gates in the previous levels have been processed. If the arrival time windows are set for all inputs of a victim gate, then this gate is pushed into a FIFO queue called the propagate frontier, which maintains the list of gates that are to be processed.

\section{The Chicken-Egg Problem}

Though we can use arrival times at the aggressor to determine whether it can switch with the victim, it is possible that this information is not available at the aggressor when required. There are two reasons why this can happen:

i) Our choice of the graph traversal method processes the victim before the aggressor.

ii) The aggressor cannot be processed before the victim because the EAT or LAT at the aggressor depends on that of the victim.

In the case of (i), one can modify the STA algorithm such that when the victim requires the EAT and LAT at the aggressor, the algorithm traces back from the aggressor to the primary input and computes them on demand. If (ii) occurs, then we encounter what we term a "Chicken-egg" problem- i.e. the aggressor and victim arrival times depend on each other. A simple example of such a case is shown in Fig. 3. If $V$ is the victim and $A$ is the aggressor, then it can be seen that the arrival time windows at $A$ would depend on those at $V$.

\section{Computing Bounds on Arrival Times}

In order to compute bounds on the arrival times (both EAT and LAT) at the primary outputs, we need to ensure that we have bounds on the EAT and LAT at each node in the

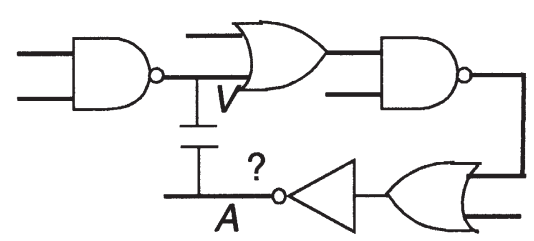

FIGURE 3 The Chicken-egg problem. 
circuit. This in turn requires that we have bounds on the BCD and WCD from each input to the gate output. When the aggressor timing window is not available, TACO uses a worst-case assumption in order to ensure that the delays computed are the absolute best and worst. To accomplish this, the EAT for all nodes is initially set to zero, and the LAT is set to infinity (or the latest possible switching time, which is the estimated clock period). We then perform one run of STA on the circuit, and compute EATs and LATs at all nodes in the circuit. Since we assume an infinite initial switching window for all nodes in the circuit, all aggressors are taken into account for their effect on delay if their windows have not been computed. This gives significant scope for pessimism in the analysis. Then, in order to overcome this pessimism, we perform a second run of STA on the circuit. During this second run, we find that some aggressors which we had assumed were aligned, are indeed temporally isolated from the victim. Hence the timing windows of such nodes would constrict and we would obtain tighter bounds for them. This reduction in windows could then propagate along various paths. We can theoretically repeat this process to further tighten the windows, but in practice we find that beyond a second iteration, the windows do not shrink significantly. This process is represented as a flowchart in Fig. 4. The pseudocode for our timing analysis algorithm which follows a block-based methodology, is shown in Fig. 5.

\section{Criterion for Aggressor Alignment}

An aggressor is said to be aligned if it is capable of contributing to the delay of the victim gate. This implies that to have an effect on delay, the window of the noise waveform at the victim fanout should overlap with the window of the victim output without noise. Depending on

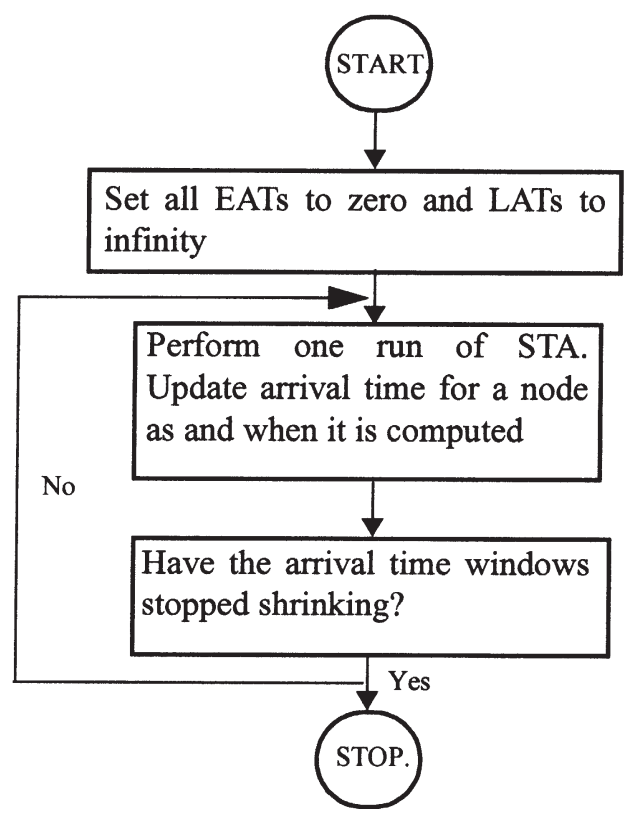

FIGURE 4 Flowchart of TACO methodology.

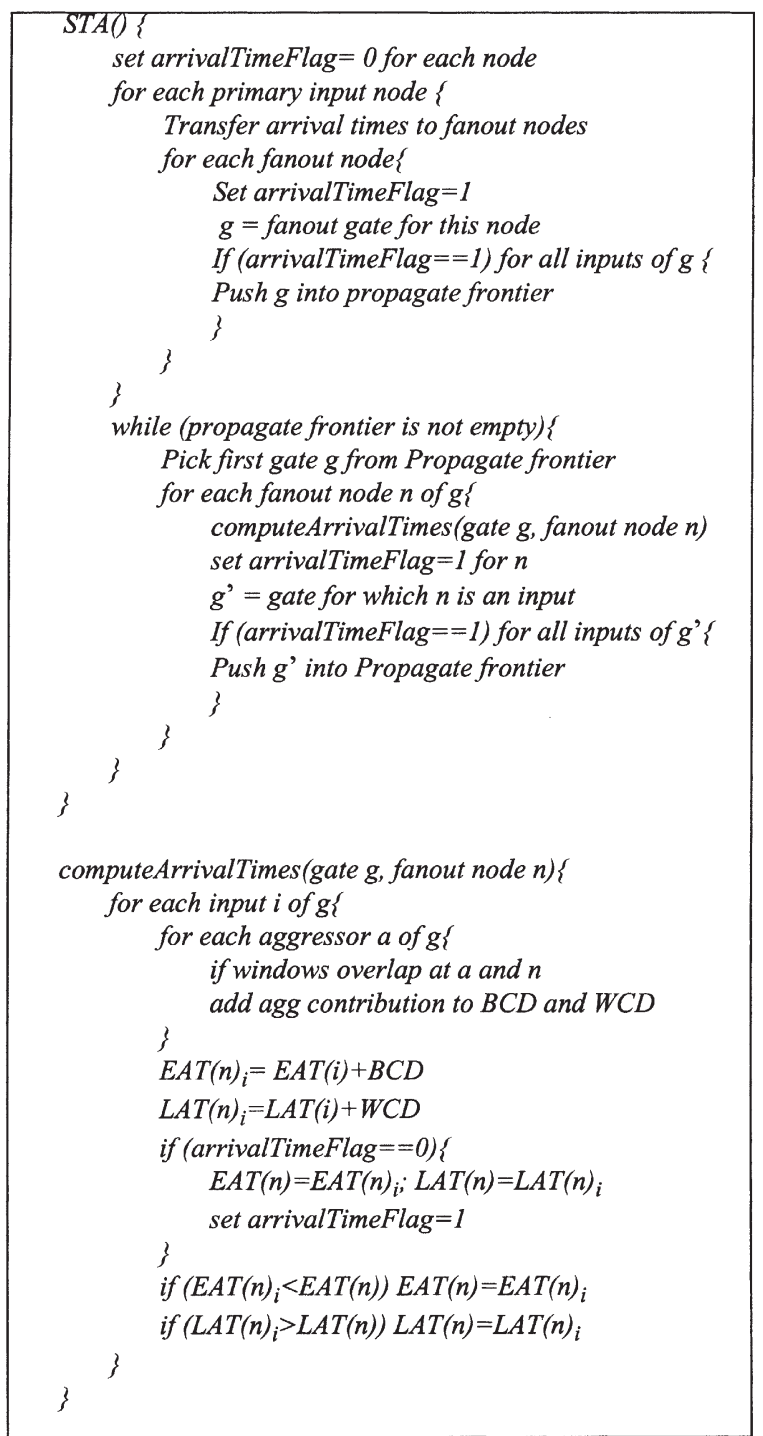

FIGURE 5 STA algorithm used in TACO.

the exactness of the analysis, a suitable condition can be used to determine if the aggressor can be aligned. The conditions we used in our analysis were as follows:

The aggressor cannot be aligned with the victim if

i) The latest noise peak time occurs before the EAT at the victim driver port.

ii) The LAT at the victim fanout occurs before the EAT at the aggressor driver port.

It should be noted that these conditions produce a pessimistic bound on the delay. The overlap criterion is shown in Fig. 6. "A" refers to the aggressor and "V" refers to the victim. The critical node where potential overlap should be checked is the fanout node and not the driver node. As shown in the figure, even though the aggressor switches later than the victim, it can create noise that affects the victim. This effect cannot be observed by using switching windows at either the input or the driver nodes of the gates. 

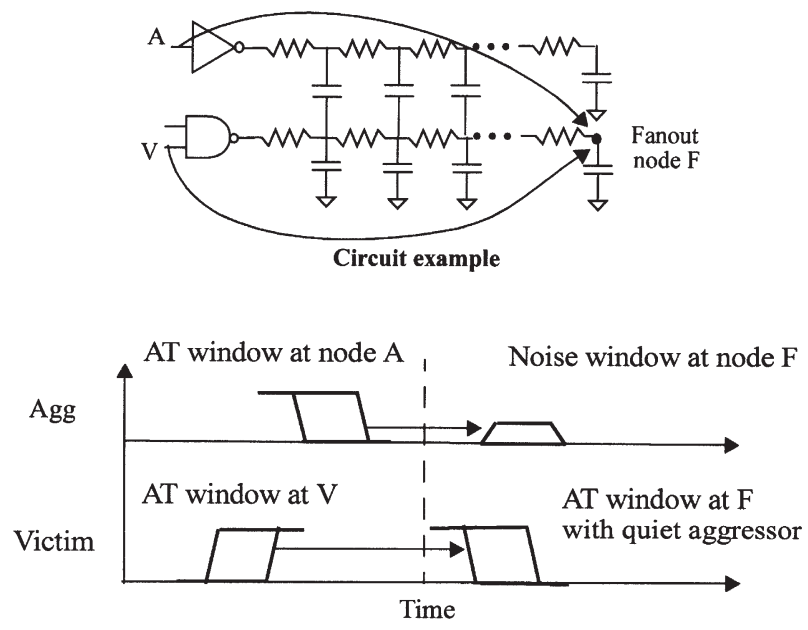

FIGURE 6 Criterion for aggressor alignment.

\section{Pruning of Aggressors}

If we consider a long global net from a typical industrial circuit, we find that it is coupled to hundreds of other nets. Most significant coupling capacitance is to same layer metal lines of which there are only a few. A large number of much smaller coupling capacitances are to nets on other metal layers. In our model, we consider as aggressors only those coupled nets, which have a coupling capacitance to total capacitance ratio greater than a certain user specified threshold. If a threshold value of between 0.15 and 0.20 is used, it is found that the number of nonnegligible aggressors is typically between 1 and 3 . Table I shows the distribution of coupled nets with change in the threshold ratio for one of the industrial examples that we considered. From this example, it can be seen that a threshold ration of $15 \%$ limits the number of aggressors nets to 3 , while not losing any significant information.

One important point to note is that there is not much accuracy lost in analyzing only these "major" coupled nets. This is because the coupling capacitance as a percentage of the total capacitance for all the other nets is typically very small. Hence, most coupled nets do not inject much noise individually. If we consider a large number of such weakly coupled nets, their effect would get statistically evened out if we consider them uncorrelated. One obvious exception to such a pruning method is when a victim net is coupled at a number of correlated lines, which might all switch at the same time in the same direction. For a more accurate analysis,

TABLE I Percentage of nets with a certain number of aggressors, as a function of threshold ratio for pruning

Number of aggressors

\begin{tabular}{lrrrrrr}
\cline { 2 - 7 } Threshold ratio $(\%)$ & \multicolumn{1}{c}{0} & \multicolumn{1}{c}{1} & 2 & 3 & 4 & $>4$ \\
\hline 5 & 3 & 7 & 27 & 28 & 22 & 13 \\
10 & 13 & 31 & 35 & 16 & 4 & 1 \\
15 & 22 & 47 & 26 & 5 & 0 & 0 \\
20 & 37 & 43 & 18 & 2 & 0 & 0
\end{tabular}

the coupling capacitance threshold can be made lower so that more aggressors are included in the analysis. On the other hand, a high threshold value can be used for a preliminary fast analysis.

\section{Slew}

The slew of a signal is indicative of how fast the signal transitions from a 0 to a 1 or vice-versa. The slew of a signal markedly affects the delay of the gate it is driving, and hence it is vital to the accuracy of timing analysis. Traditionally, the slew value has been "tied" to the arrival time value at a particular node. This means that while the EAT and LAT of the window represent the absolute earliest and latest possible times at which the node can undergo a transition, the early slew and the late slew do not necessarily represent the fastest and slowest possible rate of transitions. The difference is especially critical for coupled circuits, where the load for computing EAT and LAT can be significantly different.

We propose a new arrival time window model to handle the slew effects. We separate the slew value from the arrival time value in defining the timing window, and maintain a "slew range" for each node. The slew range represents the absolute fastest and slowest possible transitions at the particular node. With subsequent iterations, we ensure that the slowest possible slew can only become larger (i.e. the signal gets faster) and the fastest possible slew can only become smaller (i.e. the signal gets slower). Our methodology of computing the BCD and WCD combined with the way we have defined slew, makes this possible. For any gate, the fastest slew at the input is used for computing EAT at the output, and the slowest slew is used for computing LAT. The noise value is always computed using the fastest slew at the aggressor. The new models for the arrival time window and slew are shown in Fig. 7. As will be seen later, this slew model facilitates the proof of convergence for our methodology.

\section{Global Nets}

When extraction is done for a given region of the chip, there are a large number of global nets that pass through the region but do not have driver nodes and fanout nodes in the extracted circuit. We need a model to analyze these nets as some of the signal nets are coupled to global nets. We pick the port nodes of the global nets and use one as the driver node and the rest as fanout nodes. The windows of switching of the global nets is assumed to be over all time as nothing is known about them. The slew used to

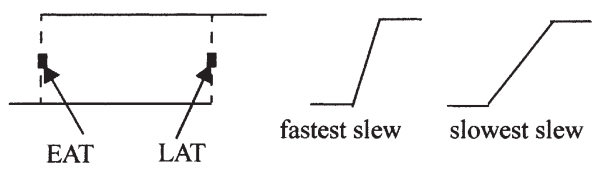

FIGURE 7 New model for arrival time windows. 


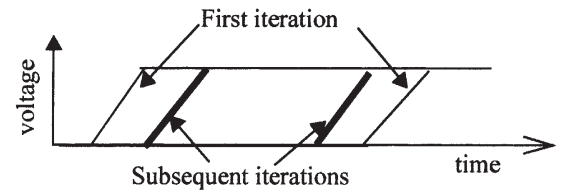

FIGURE 8 Shrinking arrival time windows.

drive the global net should typically be the fastest possible slew in the circuit. This will facilitate computation of a bound for the windows as they are primarily used as aggressors. A standard driver resistance is used for the Thevenin model of the global nets.

\section{Convergence}

The proof of convergence for our approach hinges primarily upon starting with the worst-case assumption. The timing window we obtain during the first pass is a bound on the actual timing window, for each node in the circuit. Specifically, the EAT computed during the first iteration is the absolute earliest possible for that node, and the LAT is the absolute latest possible. This is shown in Fig. 8.

Part of the proof for this is summarized in Fig. 9. If an initially aligned aggressor ceases to be aligned in a later iteration, the victim window shrinks because the WCD (BCD) will decrease (increase) without the noise contribution from the aggressor. If it continues to align and the noise contribution to delay reduces, the victim window still shrinks. There is an assumption here that the noise due to an aggressor cannot increase with subsequent iterations. Our choice for the slew model ensures this. Since we use the fastest slew possible at the aggressor during the fast iteration, it is guaranteed that the noise value does not increase with subsequent iterations.

Next we consider an aggressor that is not aligned in the first iteration. What we have shown in Fig. 9 also holds for any aggressor's timing window (since it will be treated as a victim at some point in the analysis). In conjunction with the alignment criterion defined in the "Criterion for aggressor alignment" section, and the fact that we start with the worst-case assumption, this implies that an aggressor that is not aligned in the first iteration can never do so in subsequent iterations. Hence, the timing window

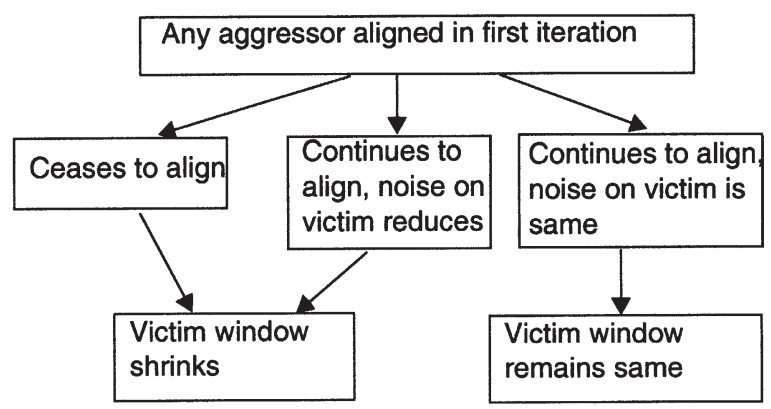

FIGURE 9 Convergence of TACO algorithm.
TABLE II Circuit data for results in "Section 3.4"

\begin{tabular}{lcc}
\hline Circuit & Number of gates & Maximum coupling cap ratio (\%) \\
\hline 1 & 126 & 70 \\
2 & 57 & 17 \\
\hline
\end{tabular}

of the victim corresponding to this aggressor remains unchanged.

It should be noted that the shrinking of victim timing windows cannot go on forever. Once the alignment status of each aggressor is established as either "aligned" or "unaligned", all timing windows have converged. In practice, we find that this status does not change after the first iteration.

\section{Results}

We ran TACO on two $0.35 \mu$ industrial examples from a microprocessor. The circuits were transistor-level netlists with coupled interconnect information. These netlists were converted to a gate level netlist using the pattern recognition tool, tranalyze [4]. Two passes of TACO were run on these circuits, and the results obtained are tabulated below. From the circuit data in Table II, it is clear that the 2 circuits span a wide range of coupling capacitance ratios, though they do not have a large number of gates. We chose these circuits because of the lack of any publicly available benchmark circuits with extracted coupling data. Table III shows data obtained by performing one run of timing analysis on these two circuits with and without the influence of aggressors. As expected, there is a significant increase in stage delay when aggressors switch in conjunction with the victim. This increase is approximately $40 \%$ for a strongly coupled gate. The change in arrival times at the primary outputs is indicative of the change in slack through the critical path in the circuit due to crosstalk. This information can be used by the designer to resize and/or resysnthesize those parts of circuit affected by crosstalk.

Figure 10 shows the distribution of increase in stage delays for all the nets analyzed with coupling. Again, the results were obtained after one pass of STA for the circuit number 1 in Table II. It can be seen that the average increase in stage delay is reflected as a similar percentage increase in the overall path delays. It should be noted that the reported crosstalk effects on delay are primarily for local signal lines and not long global wires. It is expected that the crosstalk effects on the delays through larger

TABLE III Effect of crosstalk on delay for the circuits in "Convergence" section

\begin{tabular}{lcc}
\hline Circuit & $\begin{array}{c}\text { Maximum \% increase in } \\
\text { stage delay }\end{array}$ & $\begin{array}{c}\text { Maximum \% increase in } \\
\text { path delay }\end{array}$ \\
\hline 1 & 39.5 & 12.5 \\
2 & 15.1 & 9.2 \\
\hline
\end{tabular}




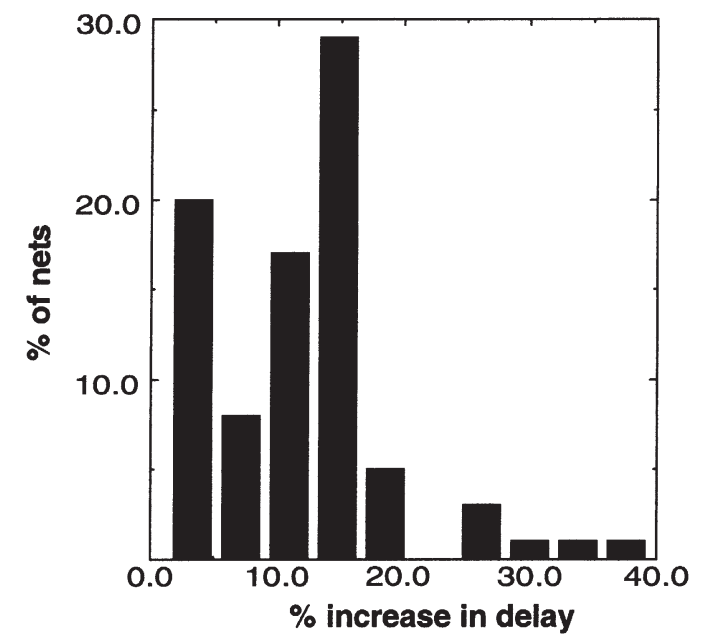

FIGURE 10 Distribution of increases in delay due to coupling.

blocks will be considerably higher because of the longer wires.

The increase in delays due to coupling shown above is after one pass of block based timing analysis. This represents the first iteration for TACO. TACO performs a second run of STA to reduce pessimism in the timing windows. Table IV shows the percentage change in the stage delays and path delays after the second run. The change in delays is primarily due to the reduction in the number of aligned aggressors. It should be noted that the resultant changes in slew also have a significant effect on the gate delays down-stream. The reduction in window size ensures that the timing windows correspond as tightly as possible to the actual windows. The pessimistic windows obtained in the first run are crucial in determining exact alignment in the second run. If we had instead performed uncoupled timing analysis, then it is possible that we would have missed some aggressors during the alignment process. These iterations could continue, but the results do not change significantly beyond the second iteration.

\section{Implementation in Einstimer}

The TACO approach was also integrated into Einstimer, a commercial timing analysis tool at IBM. It was run on a portion of a $0.5 \mu$ design with extracted coupling. The circuit was comprised of 3210 gates and a maximum coupling cap ratio of $29 \%$. The delays and arrival times obtained using the TACO methodology were compared with the simple scaling approach. Experiments were
TABLE V Comparison of TACO approach with scaling approach for the circuit mentioned in "Section 3.4.1"

\begin{tabular}{lcc}
\hline Scaling factor & $\begin{array}{c}\text { Max difference in stage } \\
\text { delay }(\%)\end{array}$ & $\begin{array}{c}\text { Max difference in path } \\
\text { delay }(\%)\end{array}$ \\
\hline 1 & +33.3 & +11.8 \\
1.5 & -34.9 & +8.2 \\
2 & -40.4 & -4.4 \\
\hline
\end{tabular}

performed with scaling factors of $1,1.5$ and 2 for the coupling capacitors, and the results obtained are tabulated in Table V. A scaling factor of 1 just means that the coupling caps were modeled as grounded.

A positive difference in the delay indicates that the WCD computed by TACO was more than that from the scaling approach, and vice-versa. A unity scaling factor is guaranteed to underestimate the WCD, as is reflected in the results. But more importantly, TACO is able to come up with a much tighter bound on delay compared to the scaling approach with a factor of 2 . Since TACO starts with a worst-case assumption for aggressor alignment, the true WCD (BCD) of the circuit cannot be greater (lesser) than the one obtained from TACO. This implies that TACO is able to reduce a significant amount of pessimism incurred due to the $2 \times$ approach. The results also indicate that while a scaling factor of 1.5 can overestimate the stage delay, it can underestimate the path delay. The impact of coupling on path delay depends on the extent of coupling along the path, the strengths of various coupled nets etc. Hence, it is impossible to use a single scaling factor for an entire design.

The run times of Einstimer on this example are as follows:

CPU time for plain scaling approach: 25.7 units.

CPU time with the TACO approach: 30.3 units.

The above run times compare one run of TACO with that of the scaling approach. It can be seen that TACO is able to obtain better bounds on the timing windows with only an $18 \%$ increase in the run time. This increase in run time is due to the complexity of coupled $C_{\text {eff }}$ iterations to accurately compute stage delay. For this example, the timing windows did not change significantly during a second iteration, and hence results are shown only for one iteration of TACO. The number of iterations required, however, is layout and design dependent.

TABLE IV Effect of a second run of STA

\begin{tabular}{lrrrr}
\hline & & & & \% Of aligned aggressors \\
\cline { 3 - 5 } Circuit & Maximum \% change in stage delay & Maximum \% change in path delays & Iteration 1 & Iteration 2 \\
\hline 1 & 26.1 & 6.6 & 50.7 & 10.7 \\
2 & 6.0 & 5.1 & 0.0 \\
\hline
\end{tabular}


Every extra iteration of TACO involves a complete run of timing analysis, and hence will increase the run time. TACO offers designers the flexibility to stop the iterations if significant reduction in pessimism is not expected. It should be noted that even the first iteration of TACO gives results that are more accurate than the scaling approach, with the $18 \%$ increase in run time. Hence, the methodology can be easily scaled to largesized circuits.

\section{FUNCTIONAL ISOLATION}

\section{Problem Formulation}

We limit the problem of finding false interactions to combinational circuits only. Sequential circuits can be separated at flip-flops and the flip-flop outputs can be treated as primary inputs, as is usually done in STA. Coupling interactions between the victim and aggressors can be categorized into two types: delay-increase and delay-decrease. The delay-increase interaction occurs when all aggressors switch in the opposite direction to that of the victim, and vice-versa. We will not deal with the noise problem caused by coupling.

We denote a rising transition on a line by $\uparrow$ and a falling transition by $\downarrow$. Since we are dealing with transitions on signal lines rather than just single values, there are two different input vectors for which the circuit needs to be analyzed. Note that this implies that we use the 2-vector transition mode [14] for analysis. This is different from the floating mode analyses in Ref. [15] whereby the initial state of the circuit is assumed to be known. We denote the two vectors by $v_{1}, v_{2}$ and the clock period of the circuit by $T . s\left(t ; v_{1}, v_{2}\right)$ represents the "state" of a particular signal, $t$ time units after vector $v_{2}$ is applied. Though the state is defined after $v_{2}$ is applied, it depends on the value of the signal before $v_{2}$, and hence $v_{1}$. Possible values of $s\left(t ; v_{1}, v_{2}\right)$ are $0,1, \uparrow, \downarrow$ for $0<t<T$ and 0,1 for $t=0$ and $t=T$. We assume the circuit reaches steady state after $v_{1}$ is applied and before $v_{2}$ is applied.

Definition A system $S$ is defined as a set of lines with one victim $V$ and $n$ aggressors $A_{1}, A_{2} \ldots A_{n}$ that are coupled to it.

$$
S=\left\{V, A_{1}, A_{2} \ldots A_{n}\right\}
$$

Definition The delay-increase interaction of a system $S=\left\{V, A_{1}, A_{2}, \ldots A_{n}\right\}$ is valid only if:

$$
\begin{gathered}
\exists v_{1}, v_{2}, t^{\prime}, 0<t^{\prime}<T, \\
V\left(t^{\prime} ; v_{1}, v_{2}\right)=\uparrow, A_{i}\left(t^{\prime} ; v_{1}, v_{2}\right)=\downarrow, i=1,2 \ldots n
\end{gathered}
$$

or

$$
V\left(t^{\prime} ; v_{1}, v_{2}\right)=\downarrow, \quad A_{i}\left(t^{\prime} ; v_{1}, v_{2}\right)=\uparrow, \quad i=1,2 \ldots n
$$

Similarly, the delay-decrease interaction is valid only if:

$$
\begin{gathered}
\exists v_{1}, v_{2}, t^{\prime}, 0<t^{\prime}<T, \\
V\left(t^{\prime} ; v_{1}, v_{2}\right)=\uparrow, A_{i}\left(t^{\prime} ; v_{1}, v_{2}\right)=\uparrow, \quad i=1,2 \ldots n
\end{gathered}
$$

or

$$
V\left(t^{\prime} ; v_{1}, v_{2}\right)=\downarrow, \quad A_{i}\left(t^{\prime} ; v_{1}, v_{2}\right)=\downarrow, \quad i=1,2 \ldots n
$$

For all aggressors to contribute to the delay of the victim, the corresponding coupling interaction must be valid. It should be noted that an interaction which is invalid for a system with one victim and $n$ aggressors can be valid for a subsystem that includes only a subset of the aggressors.

It is not necessary to compute the value of each signal for each time point $t \in[0, T]$ in order to determine whether a coupling interaction is valid. The arrival time windows can be used to determine whether a $t^{\prime}$ can possibly exist. This process of temporal pruning identifies those systems where the aggressors and victim can "potentially" switch simultaneously. It should be noted that STA can never ensure that they switch together, since it performs an input pattern independent analysis. We can now remove the simultaneity constraint from the conditions given above, which means the conditions (3) and (4) can be relaxed to:

$$
\begin{gathered}
\exists v_{1}, v_{2}, t^{\prime}, t_{i}, \quad 0<t^{\prime}<T, \quad 0<t_{i}<T, \quad i=1,2 \ldots n \\
V\left(t^{\prime} ; v_{1}, v_{2}\right)=\uparrow, A_{i}\left(t_{i} ; v_{1}, v_{2}\right)=\downarrow, \quad i=1,2 \ldots n
\end{gathered}
$$

or

$$
V\left(t^{\prime} ; v_{1}, v_{2}\right)=\downarrow, \quad A_{i}\left(t_{i} ; v_{1}, v_{2}\right)=\uparrow, \quad i=1,2 \ldots n
$$

The functional and temporal aspects of the problem are separated from each other in this manner. Even though we remove the simultaneity constraint, we only incur an error on the conservative side. If it so happens that interactions classified as valid under the above conditions cannot take place in reality, then the victim delays are overestimated. This is acceptable because we are only interested in bounds for the delays and arrival times in STA. Our goal in identifying false interactions is to still bound the actual delays while ensuring that the bounds are as tight as possible.

\section{Static Vs. Dynamic Sensitization}

DEFINITION The delay-increase coupling interaction of a system is statically sensitizable if:

$$
\begin{gathered}
\exists v_{1}, v_{2}: \\
V\left(0 ; v_{1}, v_{2}\right)=0, \quad V\left(T ; v_{1}, v_{2}\right)=1, \\
A_{i}\left(0 ; v_{1}, v_{2}\right)=1, A_{i}\left(T ; v_{1}, v_{2}\right)=0, \quad i=1,2 \ldots n
\end{gathered}
$$


or

$$
\begin{gathered}
V\left(0 ; v_{1}, v_{2}\right)=1, \quad V\left(T ; v_{1}, v_{2}\right)=0 \\
A_{i}\left(0 ; v_{1}, v_{2}\right)=0, A_{i}\left(T ; v_{1}, v_{2}\right)=1, \quad i=1,2 \ldots n
\end{gathered}
$$

A similar definition holds for the delay-decrease interaction.

Static sensitizability implies that there exist input vectors $v_{1}$ and $v_{2}$ that cause transitions on each of the victim and aggressor lines. An interaction that is not statically sensitizable is "statically unsensitizable".

THEOREM 1 If Eq. (9) holds for an input vector pair $\left(v_{1}, v_{2}\right)$, then Eq. (10) holds for the vector pair $\left(v_{2}, v_{1}\right)$.

This will reduce the number of cases that must be analyzed by half for each system.

Example 1 Consider the circuit shown in Fig. 11. Consider the delay-increase interaction with $V=\uparrow$, and $A=\downarrow$. For this interaction to be statically sensitizable, there must be a $\left(v_{1}, v_{2}\right)$, such that $V\left(T ; v_{1}, v_{2}\right)=1$ and $A\left(T ; v_{1}, v_{2}\right)=0$. Justifying these values requires $a\left(T ; v_{1}, v_{2}\right)=1, \quad e\left(T ; v_{1}, v_{2}\right)=1, \quad c\left(T ; v_{1}, v_{2}\right)=1$, $f\left(T ; v_{1}, v_{2}\right)=0$, and $A\left(T ; v_{1}, v_{2}\right)=1$. But this leads to a contradiction on $A$. Hence, the delay-increase interaction for this system is statically unsensitizable.

On the other hand, the delay-decrease interaction is sensitizable with the input vectors $v_{1}=\{0,1,1,1\}$ and $v_{2}=\{0,1,0,1\}$.

THEOREM 2 A statically sensitizable interaction is always valid.

Proof Since the state of the victim and each aggressor signal changes after $v_{2}$ is applied, they must necessarily undergo transitions in between. Hence, a statically sensitizable delay-increase interaction has to satisfy Eqs. (7) or (8). A similar argument applies for the delaydecrease interaction.

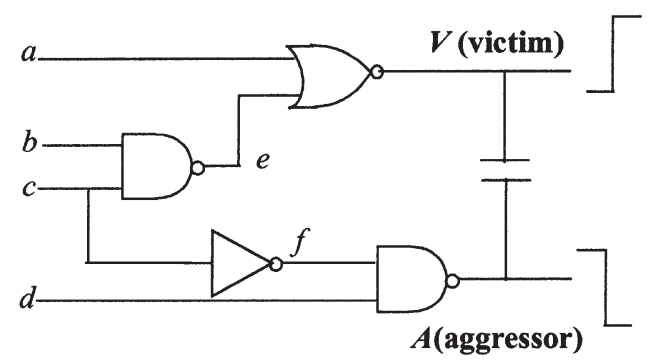

FIGURE 11 Static sensitization criterion for a coupling interaction.
DEFINITION The delay-increase interaction for a system $S$ is said to be dynamically sensitizableif:

$$
\begin{gathered}
\exists v_{1}, v_{2}: \\
V\left(0 ; v_{1}, v_{2}\right)=0, \quad V\left(T ; v_{1}, v_{2}\right)=1, \\
\exists D \subset\{1,2 \ldots n\} \quad D \neq \phi, d \in D, t_{d}, 0<t_{d}<T: \\
A_{d}\left(0 ; v_{1}, v_{2}\right)=A_{d}\left(T ; v_{1}, v_{2}\right) \neq A_{d}\left(t_{d} ; v_{1}, v_{2}\right) \\
A_{i}\left(0 ; v_{1}, v_{2}\right)=1, A_{i}\left(T ; v_{1}, v_{2}\right)=0, i \notin D
\end{gathered}
$$

or

$$
\begin{gathered}
\exists v_{1}, v_{2}: \\
V\left(T ; v_{1}, v_{2}\right)=1, V\left(T ; v_{1}, v_{2}\right)=0, \\
\exists D \subset\{1,2 \ldots n\} \quad D \neq \phi, d \in D, t_{d}, \quad 0<t_{d}<T: \\
A_{d}\left(0 ; v_{1}, v_{2}\right)=A_{d}\left(T ; v_{1}, v_{2}\right) \neq A_{d}\left(t_{d} ; v_{1}, v_{2}\right) \\
A_{i}\left(0 ; v_{1}, v_{2}\right)=0, A_{i}\left(T ; v_{1}, v_{2}\right)=1, \quad i \notin D
\end{gathered}
$$

The set $D$ contains the list of all indices $d$ such that $A_{d}$ is a "dynamic" aggressor. The signal value on a dynamic aggressor $A_{d}$ is the same at $t=0$ and $t=T$. However, because of a glitch on the line, it toggles state in between. It does not matter whether the glitch is due to a static 0hazard or a static 1-hazard. In either case, the aggressor transitions in both directions, and hence can cause a valid interaction with the victim. The condition for a dynamically sensitizable interaction is that at least one aggressor must satisfy the above property.

THEOREM 3 A dynamically sensitizable interaction is valid.

Proof Without loss of generality, we consider only the delay-increase interaction described by Eq. (11). If Eq. (11) is satisfied, then it implies that $V$ has to be $\uparrow$ at some time point. Similarly, for all $i \notin D, A_{i}=\downarrow$ at some point. For $d \in D$, since a glitch occurs on the $A_{d}$, it must have both $\downarrow$ and $\uparrow$. Hence Eq. (3) is satisfied. A similar argument holds if Eq. (12) is true.

Example 2 Consider the same circuit presented in Fig. 11, but with the delay assignments as shown in Fig. 12. Consider the input vectors $v_{1}=(0,1,1,1)$ and $v_{2}=$

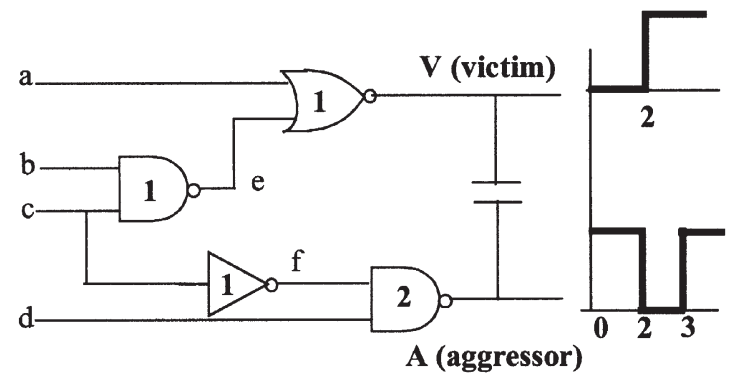

FIGURE 12 Dynamically sensitizable coupling interaction. 
TABLE VI Six-valued algebra

\begin{tabular}{ll}
\hline Signal & \multicolumn{1}{c}{ Description } \\
\hline s0 & Steady 0-signal \\
s1 & Steady 1-signal \\
p0 & Transition to 0 \\
p1 & Transition to 1 \\
g0 & Glitch on a 0 signal \\
g1 & Glitch on a 1 signal \\
\hline
\end{tabular}

$(0,1,0,0)$. If we simulate these vectors, we get:

$$
\begin{aligned}
V\left(T ; v_{1}, v_{2}\right) & =0, \quad V\left(T ; v_{1}, v_{2}\right)=1, A\left(0 ; v_{1}, v_{2}\right) \\
& =A\left(T ; v_{1}, v_{2}\right)=1 .
\end{aligned}
$$

Because of the delay assignments to the gates, however, $A\left(2 ; v_{1}, v_{2}\right)=\downarrow$ as shown in Fig. 12. The glitch on the aggressor can cause an increase in the victim's delay.

A valid coupling interaction can be either statically sensitizable or dynamically sensitizable or both. We present an ATPG-based approach to identify valid coupling interactions.

\section{ATPG Approach}

Since valid interactions can be dynamically sensitizable, any algorithm to detect valid interactions has to include glitches caused by static and dynamic hazards. We choose ATPG techniques because they can easily incorporate such elements in an algebra. We present a six-valued algebra that is used in conjunction with our ATPG approach. The values of the algebra incorporate both input vectors $v_{1}$ and $v_{2}$.

\section{Six-valued Algebra}

The elements of our algebra are shown in Table VI. Some examples of signals and how they are propagated using this algebra are shown in Fig. 13. A p0 (transition to 0) signal on a line $L$ implies that $L\left(0 ; v_{1}, v_{2}\right)=1$, and $L\left(T ; v_{1}, v_{2}\right)=0$. Similarly, a g0 signal on a line $L$ implies that $L\left(0 ; v_{1}, v_{2}\right)=0, L\left(T ; v_{1}, v_{2}\right)=0$ and that there can potentially be a glitch on the line. It should be noted that the transitions $\mathrm{p} 0$ and $\mathrm{p} 1$ can include dynamic hazards, i.e. signals that have one or more intermediate transitions as shown at the output of the XOR gate in Fig. 13. Similarly, $\mathrm{g} 0$ and g1 can include multiple glitches on a line. A value

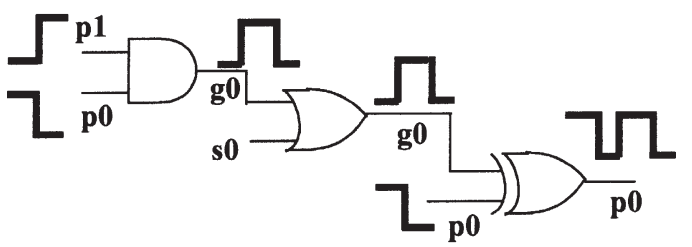

FIGURE 13 Signal propagation using the six-valued algebra. of $\mathrm{g} 0$ on a line implies that the initial and final values are the same and that there is potential for at least one glitch.

Since the effects of both $v_{1}$ and $v_{2}$ are implicitly accounted for in the algebra, one input vector in this algebra is enough to analyze a system for valid interactions. The four possible input signals that can occur at a primary input line $L$ are given in Table VII. We assume that glitches cannot occur at primary inputs. This assumption holds as long as the primary inputs are either the actual synchronous inputs to the circuit or outputs of edge-triggered flip-flops. In case they are outputs of transparent latches, they can also take the g0 and g1 values. We do not consider such cases in this work. An input vector that only includes signals from the six-valued algebra is denoted by $v_{s}$.

The six valued algebra that we use is essentially the same as the one presented in Ref. [9]. In Ref. [9], an eight-valued algebra is also presented to include dynamic hazards, and differentiate them from clean transitions. In our work, there is no necessity to make the distinction and the values $\mathrm{p} 0$ and $\mathrm{p} 1$ also include dynamic hazards. The distinction is required only if justification of the values on the victim or aggressor forced a primary input to a dynamic transition. In such a case, because of the constraint that primary inputs can only have clean transitions, the particular interaction would be classified as invalid, whereas without the distinction it would be considered valid. We claim that this possibility can never occur. A forced dynamic transition will be required at an input of a gate only if the output is forced to be a dynamic transition. However, the conditions necessary for static or dynamic sensitization only specify that a transition has to occur on a victim or an aggressor. They do not specify whether the transition has to be clean or give a limit on the number of transitions.

It is important to show that the algebra we use is closed with respect to Boolean operations. In addition to the six values in the algebra, it becomes necessary to use the $X$ value during ATPG, where $X$ represents an unknown value for a line. For proving closure, it suffices to show that the algebra is closed with respect to the AND, OR, and NOT operators. Closure for the NOT operator is trivial. The input-output relationships for the AND operator are given in Table VIII. It can be observed that the AND operation between any two signals in the algebra produces a signal within the algebra. The OR operator can be dealt with in a similar manner.

TABLE VII Possible combination of values that can be assigned to a primary input $L$

\begin{tabular}{lcc}
\hline$L\left(0 ; v_{1}, v_{2}\right)$ & $L\left(T ; v_{1}, v_{2}\right)$ & Signal name \\
\hline 0 & 0 & $\mathrm{~s} 0$ \\
1 & 1 & $\mathrm{~s} 1$ \\
1 & 0 & $\mathrm{p} 0$ \\
0 & 1 & $\mathrm{p} 1$ \\
\hline
\end{tabular}


TABLE VIII AND operation on signal values

\begin{tabular}{llllllll}
\hline AND & s0 & s1 & p0 & p1 & g0 & g1 & X \\
\hline s0 & s0 & s0 & s0 & s0 & s0 & s0 & s0 \\
s1 & s0 & s1 & p0 & p1 & g0 & g1 & X \\
p0 & s0 & p0 & p0 & g0 & g0 & p0 & X \\
p1 & s0 & p1 & g0 & p1 & g0 & p1 & X \\
g0 & s0 & g0 & g0 & g0 & g0 & g0 & X \\
g1 & s0 & g1 & p0 & p1 & g0 & g1 & X \\
X & s0 & X & X & X & X & X & X \\
\hline
\end{tabular}

We can now restate the conditions for static and dynamic sensitization in terms of the algebra of Table VI.

THEOREM 4 A delay-increase interaction for a system is statically sensitizable if

$$
\exists v_{s}: \quad V=\mathrm{p} 0, \quad A_{i}=\mathrm{p} 1, \quad i=1,2 \ldots n
$$

It can be seen that the condition given above is the same as Eq. (9) in the definition of a statically sensitizable interaction given in the "Static vs. dynamic sensitization" section. Since we proved via theorem 1 that Eqs. (9) and (10) are equivalent, we need not find a vector to satisfy Eq. (10) separately.

THEOREM 5 A delay-increase interaction for a system is dynamically sensitizable if

$$
\exists v_{s}: \quad V=\mathrm{p} 0, A_{i} \in\{\mathrm{p} 1, \mathrm{~g} 0, \mathrm{~g} 1\}, \quad i=1,2 \ldots n
$$

or

$$
\exists v_{s}: \quad V=\mathrm{p} 1, A_{i} \in\{\mathrm{p} 0, \mathrm{~g} 0, \mathrm{~g} 1\}, \quad i=1,2 \ldots n
$$

Again, under the definition of our six-valued algebra, Eq. (14) is equivalent to Eq. (11), and Eq. (15) is equivalent to Eq. (12).

Theorem 6 If there exists a $v_{s}$ that satisfies Eq. (14), then there exists a $v_{s}^{\prime}$ that satisfies Eq. (15).

Proof Consider the vector $v_{s}^{\prime}$ which has the same values as $v_{s}$ except for the following changes: (i) a primary input with $\mathrm{p} 0$ in $v_{s}$ is replaced by $\mathrm{p} 1$ in $v_{s}^{\prime}$ and (ii) a primary input with $\mathrm{p} 1$ in $v_{s}$ is replaced by $\mathrm{p} 0$ in $v_{s}^{\prime}$. Primary inputs with $\mathrm{s} 0$ or $\mathrm{s} 1$ in $v_{s}$ remain unchanged. Under these conditions, the following properties can be observed.

LEMMA 1 For any line $L, L\left(v_{s}^{\prime}\right)=\mathrm{p} 0(\mathrm{p} 1)$ if $L\left(v_{s}\right)=$ $\mathrm{p} 1(\mathrm{p} 0)$.

Since the initial and final values in $v_{s}$ and $v_{s}^{\prime}$ are interchanged for each primary input, the steady-state values for each line will also get interchanged under $v_{s}$ and $v_{s}^{\prime}$.

LEMMA 2 For any line $L, L\left(v_{s}^{\prime}\right)=\mathrm{s} 0(\mathrm{~s} 1)$ if $L\left(v_{s}\right)=$ $\mathrm{s} 0(\mathrm{~s} 1)$.

If $L$ is a primary input, then by our choice of $v_{s}^{\prime}$, lemma 2 holds. Suppose $L\left(v_{s}\right)$ is a stable value and is the output of gate $G$. We observe that for the output of $G$ to have a stable value ( $\mathrm{s} 0$ or $\mathrm{s} 1$ ), at least one of it's inputs must be stable. Moreover, if $L\left(v_{s}\right) \neq L\left(v_{s}^{\prime}\right)$, then at least one of those stable inputs must change values between $v_{s}$ and $v_{s}^{\prime}$. This can be rigorously proven by considering all possible inputs for the three basic gates: NOT, AND, and OR. Hence, if $L\left(v_{s}\right) \neq L\left(v_{s}^{\prime}\right)$, then there exists an input $I$ of $G$ such that $I\left(v_{s}\right) \neq I\left(v_{s}^{\prime}\right)$ and $I\left(v_{s}\right)$ is a stable value. This process of back tracing can continue till we reach a primary input, but by our choice of $v_{s}^{\prime}$, stable primary inputs have the same values in $v_{s}$ and $v_{s}^{\prime}$. This implies that $L\left(v_{s}\right)=L\left(v_{s}^{\prime}\right)$.

Lemma 3 If $A_{i}\left(v_{s}\right) \in\{\mathrm{g} 0, \mathrm{~g} 1\}, A_{i}\left(v_{s}^{\prime}\right) \in\{\mathrm{g} 0, \mathrm{~g} 1\}$.

If $A_{i}\left(v_{s}^{\prime}\right) \in\{\mathrm{s} 0, \mathrm{~s} 1, \mathrm{p} 0, \mathrm{p} 1\}$, we would get a contradiction for $A_{i}\left(v_{s}\right)$ based on lemma 1 and 2 . But we know that the six-valued algebra is closed, hence, all signals have to belong to $\{\mathrm{s} 0, \mathrm{~s} 1, \mathrm{p} 0, \mathrm{p} 1, \mathrm{~g} 0, \mathrm{~g} 1\}$. Hence, $A_{i}\left(v_{s}^{\prime}\right) \in$ $\{\mathrm{g} 0, \mathrm{~g} 1\}$.

From the above three lemmas, it follows that $v_{s}^{\prime}$ satisfies Eq. (15).

Theorem 6 is quite powerful because it reduces by half the number of cases to be analyzed for dynamic sensitization too.

\section{Choice of ATPG Algorithm}

The factors that guide our choice of an algorithm are different from those that determine the best approach for a typical ATPG application. The goal of ATPG is to find a test vector to detect a particular fault, or in other words find an input vector that justifies certain values on certain signal lines. For the problem of identifying false interactions, it is more useful to show that no vector exists that can justify the required values at the victim and the aggressor. The test vector itself is not of any practical use. Since we use a multi-valued algebra, an output value usually can be obtained from a variety of input combinations. Hence, exploring the search space by starting from the victim and aggressor lines, as in the Dalgorithm [16], turns out to be inefficient. We used a modified version of the PODEM [12] algorithm for our approach, since the search space explored in PODEM is the primary inputs. Another reason for choosing PODEM was that the primary inputs have a constraint that they can assume only a subset of values from the six-valued algebra. By its very nature, PODEM avoids conflicts at primary inputs and hence is a suitable candidate for analyzing system interactions.

There is, however, a serious limitation of PODEM in the presence of multiple objectives. If one objective causes a local conflict with another objective, then PODEM would take a long time to detect it, since it will have to exhaust the search space at the primary inputs. We implemented an imply-and-check routine to detect such obvious conflicts since our primary goal is to detect invalid coupling interactions as quickly as possible. Given a set of objectives, our approach identifies an input value using a modified version of PODEM for multiple objectives. Our algorithm is summarized in Fig. 14. The reorder function 


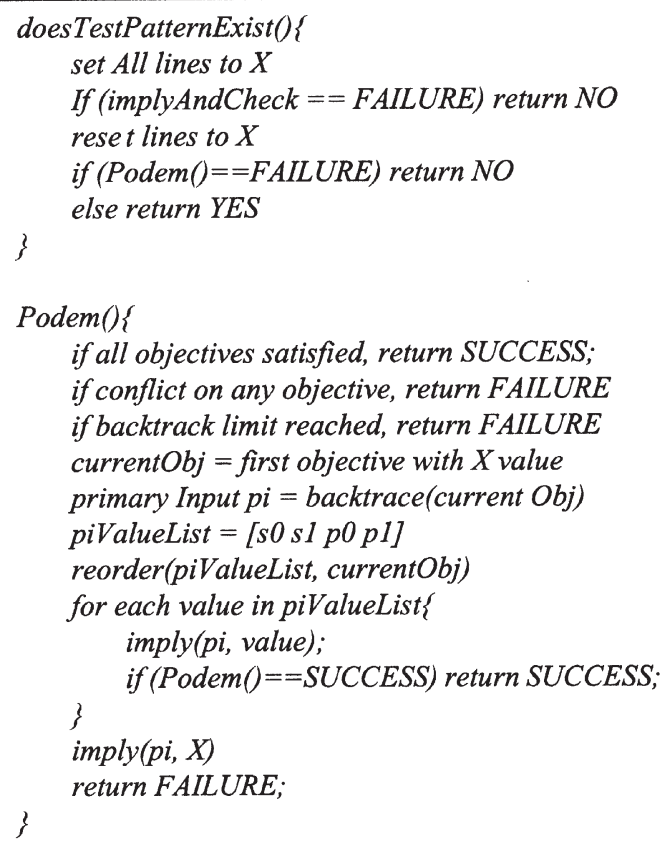

FIGURE 14 Modified PODEM algorithm to identify valid coupling interactions.

is used to determine the order of selecting primary input values based on the current objective as well as the number of inversions encountered while backtracing.

\section{Results}

Since we did not have real circuits with coupling as well as functional information, we used the ISCAS85 benchmarks [12] and selected random coupled systems from these netlists. Up to five aggressors were allowed, since the delay impact of each aggressor is very small if the number of aggressors is large. Circuit data for the ISCAS85 benchmarks is given in Table IX. The difference between the levels of any two lines in a coupled system was limited, to simulate real-circuit behavior by localizing the coupling. For each circuit, we generated 100 coupled systems and studied whether the delay-increase and delaydecrease interaction for each system was sensitizable.

TABLE IX Data for ISCAS85 benchmark circuits

\begin{tabular}{|c|c|c|c|}
\hline Circuit & No. of gates & No. of nets & $\begin{array}{c}\text { Average No. of aggressors per } \\
\text { net }\end{array}$ \\
\hline $\mathrm{C} 432$ & 160 & 196 & 3.18 \\
\hline C499 & 202 & 243 & 2.95 \\
\hline $\mathrm{C} 880$ & 383 & 443 & 3.04 \\
\hline $\mathrm{C} 1355$ & 546 & 587 & 2.99 \\
\hline C1908 & 880 & 913 & 3.07 \\
\hline C2670 & 1193 & 1426 & 3.22 \\
\hline C3540 & 1669 & 1719 & 2.86 \\
\hline C5315 & 2307 & 2481 & 3.13 \\
\hline C6288 & 2406 & 2438 & 2.94 \\
\hline C7552 & 3512 & 3719 & 3.22 \\
\hline
\end{tabular}

Since both types of interaction have to be studied independently, we had 200 sets of objectives. The number of statically sensitizable and dynamically sensitizable interactions were calculated separately. The total number of valid and invalid interactions are also reported. The results are tabulated in Table $\mathrm{X}$. The run times reported are on a Pentium III $500 \mathrm{MHz}$ PC running Linux.

It can be observed from the table that the number of statically sensitizable interactions is typically less than the number of valid interactions. This implies that a portion of valid interactions are only dynamically sensitizable. Static sensitization alone, without incorporating glitches, will lead to optimistic results, which is unacceptable in timing analysis. Our method of incorporating hazards and detecting valid interactions helps in identifying false interactions more accurately.

Since we account for all possible dynamic switchings, including both static and dynamic hazards, our approach is guaranteed to be conservative. This means that in any interaction that we claim as invalid, the victim and aggressors can never all switch together. The largest number of invalid interactions, 12 out of the total of 200, is observed for C1908. These false interactions could then be input to the static timing analyzer, which could then ignore these interactions when analyzing the coupling delay. The number of invalid interactions is observed to be relatively small for the other circuits. The results show that this number is largely design dependent and hence functional analysis is critical for eliminating conservatism. The run time for the functional analysis is circuit-dependent, and is observed to be lower for circuits with a small number of levels or low reconvergent fanout.

\section{CONCLUSIONS}

We have described a timing analysis methodology TACO that can accurately bound the arrival times in the presence of coupling. The timing analysis flow in TACO is designed such that the timing windows at the aggressor can be used to determine whether the aggressor can switch in conjunction with the victim. If it can, TACO uses the coupled $C_{\text {eff }}$ delay engine to compute best-case and worstcase stage delays. The arrival time windows along different paths may be dependent on each other, and we start with provable bounds on the delays and arrival times. This might lead to conservative results on the arrival time windows and TACO uses an iterative methodology to reduce this conservatism. We have also presented a novel slew model that uses a min/max range of slew values to represent the signal transition times at each node. We have shown convergence of our iterative methodology using the new slew modeling approach. Results on industrial examples show that the popular scaling factor approach can lead to large inaccuracies and can be unpredictable in it's accuracy. We have also demonstrated that the iterative methodology actually helps in reducing pessimism in the analysis. 
TABLE X Distribution of coupling interactions for ISCAS85 benchmarks

\begin{tabular}{|c|c|c|c|c|c|c|}
\hline Circuit & Statically sensitizable & Dynamically sensitizable & Reached backtrack limit & Valid interactions & Invalid interactions & CPU time (s) \\
\hline $\mathrm{C} 432$ & 161 & 127 & 17 & 183 & 0 & 89.3 \\
\hline C499 & 152 & 152 & 48 & 152 & 0 & 16.4 \\
\hline $\mathrm{C} 880$ & 178 & 168 & 4 & 188 & 4 & 40.3 \\
\hline $\mathrm{C} 1355$ & 194 & 196 & 0 & 198 & 2 & 5.1 \\
\hline C1908 & 188 & 50 & 0 & 188 & 12 & 2.9 \\
\hline $\mathrm{C} 2670$ & 184 & 152 & 2 & 194 & 4 & 31.9 \\
\hline C3540 & 146 & 166 & 0 & 194 & 6 & 186 \\
\hline C5315 & 198 & 146 & 0 & 200 & 0 & 15.8 \\
\hline C6288 & 178 & 176 & 0 & 194 & 6 & 5.8 \\
\hline C7552 & 182 & 160 & 0 & 192 & 8 & 17.9 \\
\hline
\end{tabular}

We have also presented a comprehensive approach to incorporate functional information in an STA flow. When a victim and set of aggressors cannot all switch simultaneously, we classify that particular interaction as a false coupling interaction, and as a valid interaction otherwise. Valid interactions are further divided into statically and dynamically sensitizable interactions, depending on the transitions on aggressors and the victim. We have further shown that static sensitization criteria alone are insufficient to identify false interactions. Glitches on aggressors, due to hazards in the circuit, can affect the victim's delay even though static sensitization might predict otherwise. We have derived the conditions under which such dynamic interactions can occur, and represented them using a multi-valued algebra. We have presented an efficient ATPG approach using this algebra to identify valid and invalid interactions. The results show the value of considering functional information to identify and eliminate pessimism in STA. Since we use a conservative approach, an interaction that is classified as invalid is guaranteed to not occur in reality.

\section{References}

[1] Dartu, F. and Pileggi, L.T. (1997) "Calculating worst-case gate delays due to dominant capacitance coupling", Proc. Design Automation Conf., 576-580.

[2] Arunachalam, R., Dartu, F. and Pileggi, L.T. (1997) "CMOS gate delay models for general RLC loading", Proc. Int. Conf. Comput. Des., 224-229.

[3] Gross, P., Arunachalam, R., Rajagopal, K. and Pileggi, L.T. (1998) "Determination of worst case aggressor alignment for delay calculation", Proc. Int. Conf. Comput.-Aided Des. November.

[4] Bryant, R.E. (1991) "Extraction of gate level models from transistor circuits by four-valued symbolic analysis", Proc. Int. Conf. Comput. Aided Des.

[5] Kirkpatrick, D. and Sangiovanni-Vincentelli, A.L. (1996) "Digital sensitivity: predicting signal interaction using functional analysis", Proc. Int. Conf. Comput.-Aided Des., 536-541.

[6] Franzini, B., Forzan, C., Pandini, D., Scandolara, P. and Fabbro, A.D. (2000) "Crosstalk aware static timing analysis: a two step approach", Proc. 1st Int. Symp. Quality Electronic Des., 499-503.

[7] Chen, P., Kirkpatrick, D.A. and Keutzer, K. (2000) "Miller factor for gate-level coupling delay calculation", Proc. Int. Conf. Comput.Aided Des., 68-73.

[8] The ISCAS-85 Benchmarks. http://zodiac.cbl.ncsu.edu/CBL_Docs/ iscas $85 . h t m l$

[9] Hayes, J.P. (1986) "Digital simulation with multiple logic values", IEEE Trans. Comput.-Aided Des. CAD-5, 274-328, April.
[10] Odabasioglu, A., Celik, M. and T.P., Lawrence (1997) "PRIMA: passive reduced-order inter-connect macromodeling algorithm", Proc. Int. Conf. Comput.-Aided Des..

[11] Chen, P., Kirkpatrick, D. and Keutzer, K. (2000) "Switching window computation for static timing analysis in presence of crosstalk noise", Proc. Int. Conf. Comput.-Aided Des., 331-336.

[12] Goel, P. (1991) "An implicit enumeration algorithm to generate tests for combinational logic circuits", IEEE Trans. Comput., 211-222, March.

[13] Kahng, A.B., Muddu, S. and Sarto, E. (2000) "On switch factor based analysis of coupled RC interconnects", Proc. Des. Automation Conf., 79-84.

[14] Devadas, S., Keutzer, K., Malik, S. and Wang, A. (1992) "Certified timing verification and the transition delay of a logic circuit", Proc. Des. Automation Conf., 549-555.

[15] Chen, H.C. and Du, D.H.C. (1993) "Path sensitization in critical path problem", IEEE Trans. Comput.-Aided Des. 12, 196-207, February.

[16] Roth, J.P. (1966) "Diagnosis of automata failures: a calculus and a method", IBM J. Res. Dev. 10(4), 278-291.

Ravishankar Arunachalam is an advisory engineer with IBM Corporation in Austin. He received the Bachelor of Technology degree in Electrical and Electronics engineering from the Indian Institute of Technology, Madras in 1996, and his M.S. and Ph.D. in Electrical and Computer Engineering, from Carnegie Mellon University in 1998 and 2000, respectively. His research interests include circuit design automation, in the areas of signal integrity, timing analysis, delay modeling and interconnect analysis. He was a recipient of the IBM fellowship for students for three consecutive years from 1997 to 2000.

Lawrence Pileggi is a professor of electrical and computer engineering and Director of the Center for Silicon System Implementation at Carnegie Mellon University. From 1984 to 1986, he worked for Westinghouse Research and Development where in 1986 he was recognized with the corporation's highest engineering achievement award. In 1989, he received his Ph.D. in Electrical and Computer Engineering from the Carnegie Mellon University. From 1989 to 1995, he was a faculty member at the University of Texas at Austin. In January of 1996, he joined the faculty at Carnegie Mellon University. His research interests include various aspects of circuitlevel design automation and analysis. He has consulted for several EDA and semiconductor companies and while on leave from Carnegie Mellon from July 1999 through July 2000 he was the CTO and VP of R\&D at Monterey Design 
Systems. He received the Best CAD Transactions Paper Award in 1991 for "Asymptotic Waveform Evaluation (AWE)," and again in 1999 for "Passive Reduced-order Interconnect Macromodeling Algorithm (PRIMA)." He received a Presidential Young Investigator Award from the National Science Foundation in 1991. In 1991 and in 1999, he received the Semiconductor Research Corporation Technical Excellence Award. In 1993, he received an Invention Award from the SRC and subsequently a U.S. Patent for the RICE simulation tool. In 1994, he received The University of Texas Parent's Association Centennial Teaching Fellowship for excellence in undergraduate instruction. In 1995, he received a Faculty Partnership Award from IBM. He is a co-author of "Electronic Circuit and System Simulation Methods," McGraw-Hill, 1995. He has published over 125 refereed conference and journal papers and holds five U.S. patents.
Shawn Blanton is an associate professor in the Department of Electrical and Computer Engineering at Carnegie Mellon University where he is a member of the Center for Silicon Systems Implementation (CSSI). He received the Bachelor's degree in engineering from Calvin College in 1987, a Master's degree in Electrical Engineering in 1989 from the University of Arizona and a Ph.D. degree in Computer Science and Engineering from the University of Michigan, Ann Arbor in 1995. His research interests include the computer-aided design of VLSI circuits and systems; fault-tolerant computing and diagnosis; verification and testing; and computer architecture. He has worked on the design and test of integrated systems with General Motors Research Laboratories, AT\&T Bell Laboratories, Intel and Motorola. Dr Blanton is the recipient of National Science Foundation Career Award and is a member of IEEE and ACM. 

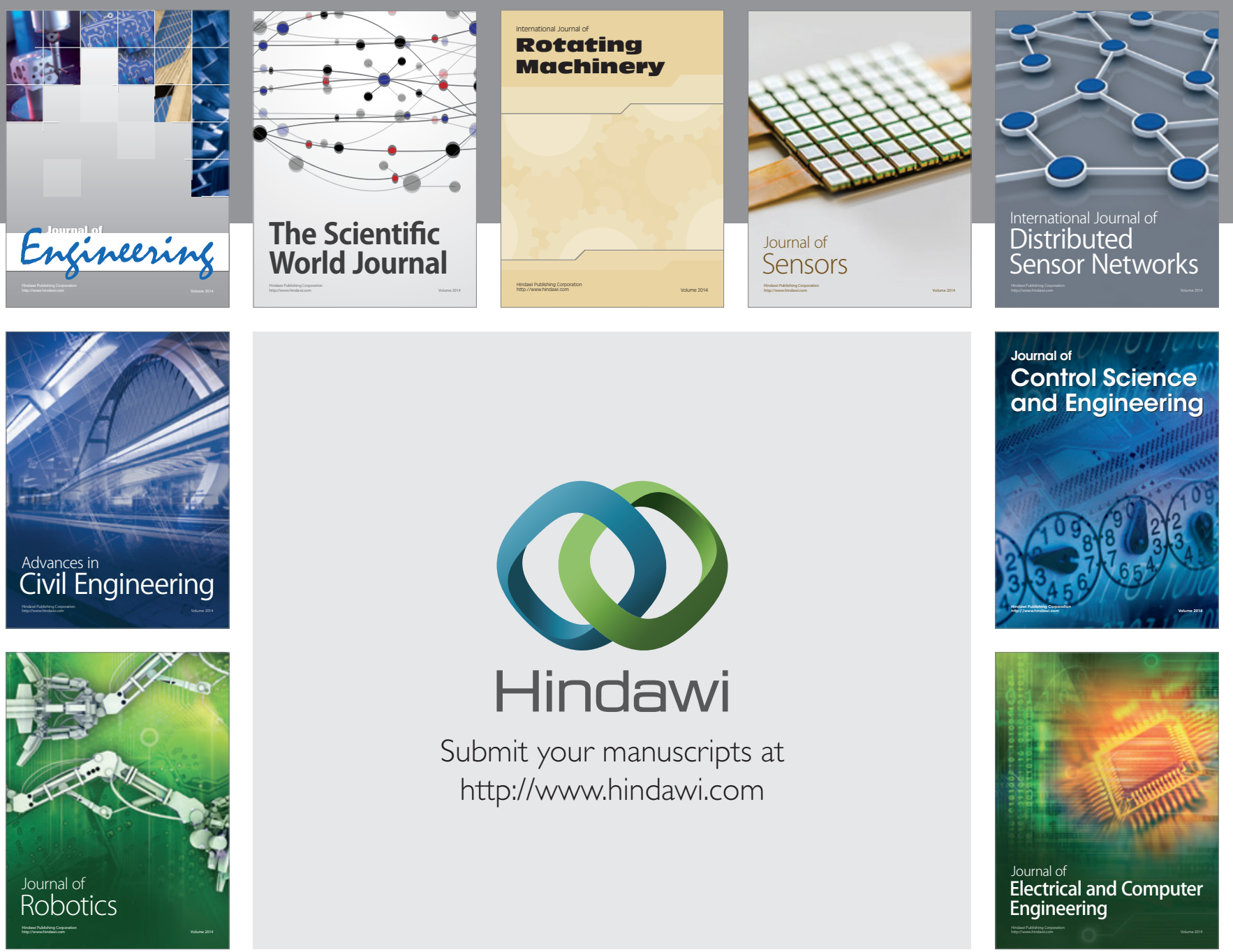

Submit your manuscripts at

http://www.hindawi.com
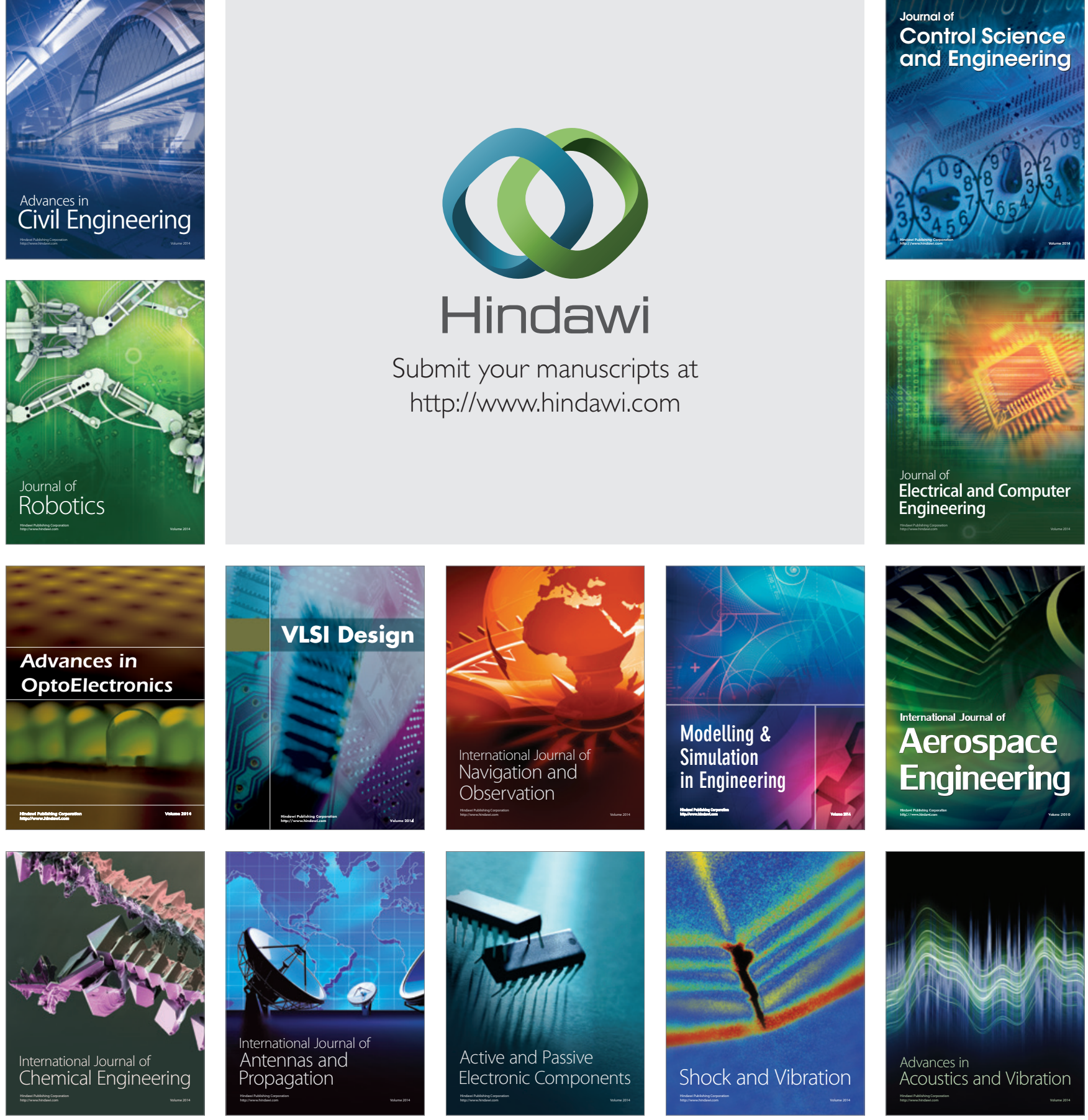